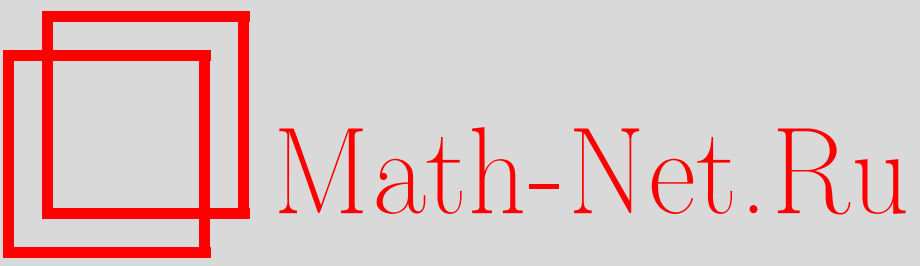

В. Р. Крым, Уравнения геодезических для заряженной частицы в объединенной теории гравитационных и электромагнитных взаимодействий, ТМФ, 1999, том 119, номер 3, 517-528

DOI: https://doi.org/10.4213/tmf755

Использование Общероссийского математического портала Math-Net.Ru подразумевает, что вы прочитали и согласны с пользовательским соглашением

http: //www . mathnet.ru/rus/agreement

Параметры загрузки:

IP : 54.237 .206 .68

26 апреля 2023 г., 15:35:41 
ТЕОРЕТИЧЕСКАЯ

И МАТЕМАТИЧЕСКАЯ

ФИЗИКА

Том 119, № 3

июнь, 1999

(C) 1999 г.

В. Р. Крым*

\title{
УРАВНЕНИЯ ГЕОДЕЗИЧЕСКИХ ДЛЯ ЗАРЯЖЕННОЙ ЧАСТИЦЫ В ОБЪЕДИНЕННОЙ ТЕОРИИ ГРАВИТАЦИОННЫХ И ЭЛЕКТРОМАГНИТНЫХ ВЗАИМОДЕЙСТВИЙ
}

\begin{abstract}
Предлагается новая модель гравитационных и электромагнитных взаимодействий, являющаяся вариантом классической теории Калуцы-Клейна и основанная на использовании пятимерного многообразия как физического пространства-времени. В нашей модели пространство скоростей движущихся частиц остается четырехмерным, как в обычной теории относительности. Пространства скоростей частиц образуют четырехмерное распределение на пятимерном гладком многообразии. Это распределение зависит только от электромагнитного поля и не зависит от поля метрического тензора. Доказано, что уравнения геодезических, таких что поле их вектора скорости всегда принадлежит указанному распределению, совпадают с уравнениями движения заряженной частицы в общей теории относительности. Калибровочные преобразования получают наглядную интерпретацию как частный вид преобразований координат на пятимерном многообразии.
\end{abstract}

\section{1. ВВЕДЕНИЕ}

Неголономная риманова геометрия, известная также как субриманова геометрия или теория пространств Карно-Каратеодори, интенсивно развивается уже в течение 15 лет [1]. Однако изучались только пространства с положительно-определенной (римановой) метрикой, и никаких приложений к общей теории относительности предложено не было. В то же время неголономная псевдориманова геометрия является наиболее естественным обобщением псевдоримановых многообразий.

Напомним, что на гладком многообразии $M$ распределением размерности $n$ называется непрерывно зависяшее от точки $x \in M$ поле $n$-мерных линейных подпространств $\mathbf{A}(x) \subset T_{x} M$. Распределение называется голономным (интегрируемым), если оно является полем касательных пространств к некоторому подмногообразию $N \subset M$. Связность на гладком многообразии - это распределение во втором касательном расслоении. В расслоенных пространствах связность является распределением в (первом) касательном расслоении к расслоенному пространству.

\footnotetext{
* Санкт-Петербургский государственный университет, Санкт-Петербург, Россия
} 
В данной работе мы рассматриваем пятимерное гладкое многообразие $M$ и четырехмерное распределение $\mathbf{A}$ на $M$, заданное ковекторным полем нормали:

$$
n:=A_{0} d x^{0}+A_{1} d x^{1}+A_{2} d x^{2}+A_{3} d x^{3}+d x^{4} .
$$

Мы увидим в дальнейшем, что координаты ковектора (дифференциальной формы) $n$ можно отождествить с 4-потенциалом электромагнитного поля. Скорость света принята равной единице, поэтому единицей измерения всех координат $A_{k}$ является вольт, координат $x^{k}$-метр, $k=0, \ldots, 3$, а пятой координаты $x^{4}-\mathrm{B} \cdot$ м. Это распределение можно также задать с помошью базисных векторных полей $e_{k}:=\partial_{k}-A_{k} \partial_{4}, k=0, \ldots, 3$, где

$$
\partial_{j}:=\frac{\partial}{\partial x^{j}}, \quad j=0, \ldots, 4,
$$

- координатные векторные поля. Инвариантность такого выбора (по отношению к специальной группе преобразований координат [2]) будет проверена в разделе 3.

Предположим, что в касательном расслоении $T M$ задано индефинитное скалярное произведение $\langle\cdot, \cdot\rangle$. Тогда можно рассмотреть сужение скалярного произведения $\langle\cdot, \cdot\rangle$ на распределение $\mathbf{A}$. Обозначим через $g_{k l}:=\left\langle e_{k}, e_{l}\right\rangle, k, l=0, \ldots, 3$, координаты метрического тензора распределения А. Мы предполагаем, что метрика на распределении $\mathbf{A}$ псевдориманова, т.е. матрища $\left(g_{k l}\right)_{k, l=0, \ldots, 3}$ имеет сигнатуру $(+---)$. Легко выписать метрику на многообразии $M$, которая будет удовлетворять этому свойству, например

$$
\bar{g}=\left(\begin{array}{cc}
\left(g_{i j}\right)_{i, j=0, \ldots, 3} & \left(-\kappa A_{j}\right)_{j=0, \ldots, 3} \\
\left(-\kappa A_{j}\right)_{j=0, \ldots, 3}^{T} & -2 \kappa
\end{array}\right) .
$$

Константа $\kappa$ введена из физических соображений, и ее значение пока не фиксировано. Поскольку матрица проекции на распределение $\mathbf{A}$ имеет вид

$$
P=\left(I_{4} \mid\left(-A_{j}\right)_{j=0, \ldots, 3}\right),
$$

где $I_{4}$ - единичная матрица порядка 4 , получаем

$$
P\left(\begin{array}{cc}
\left(g_{i j}\right)_{i, j=0, \ldots, 3} & \left(-\kappa A_{j}\right)_{j=0, \ldots, 3} \\
\left(-\kappa A_{j}\right)_{j=0, \ldots, 3}^{T} & -2 \kappa
\end{array}\right) P^{T}=\left(g_{i j}\right)_{i, j=0, \ldots, 3} .
$$

Метрический тензор $\bar{g}$ с таким свойством не единствен. С помошью математического аппарата теории оптимального управления [3] можно показать, что уравнения движения в соответствуюшей неголономной задаче не зависят от метрики в $T M$, а зависят только от метрики на распределении $\mathbf{A}$. Мы увидим это на конкретном примере. 


\section{2. НАВОДЯЩИЕ СООБРАЖЕНИЯ}

ОПреДЕЛЕниЕ. Гладкий путь $\gamma: I \rightarrow M$ называется допустимым, если $\forall t \in I$ $\gamma^{\prime}(t) \in \mathbf{A}(\gamma(t))$.

Выведем уравнения допустимых геодезических. Основываясь на аналогии с обшей теорией относительности, мы должны искать длиннейшие ${ }^{1)}$ кривые среди всех кривых, соединяюших две заданные точки. Мы не будем рассматривать вопросы сушествования таких кривых. Первый контрпример к "теореме существования" таких кривых был построен Петровым [4] для размерности 3 , см. также [5, 6]. Мы будем решать задачу на экстремум функционала длины на множестве гладких путей при дополнительном условии: путь должен быть допустимым. Получаем функционал действия

$$
S(\gamma, \lambda):=m \int_{I}\left\langle\gamma^{\prime}(t), \gamma^{\prime}(t)\right\rangle^{1 / 2} d t+\int_{I} \lambda(t) n(\gamma(t)) \cdot \gamma^{\prime}(t) d t
$$

где $m$ - фиксированная масса частицы, $\lambda: I \rightarrow \mathbf{R}$ - множитель Лагранжа. Будем считать распределение $\mathbf{A}$ гладким. Поскольку оба интеграла не зависят от параметризации пути $\gamma$, считаем, что $\gamma$ параметризован длиной дуги. В координатной записи имеем функцию Лагранжа

$$
L=m\left(\sum_{i, j=0}^{4} \bar{g}_{i j} u^{i} u^{j}\right)^{1 / 2}+\lambda\left(\sum_{i=0}^{3} A_{i} u^{i}+u^{4}\right),
$$

где $\left(u^{k}\right)_{k=0, \ldots, 4}$ - координаты вектора $\gamma^{\prime}$. Уравнения Лагранжа имеют вид

$$
m\left(\sum_{j=0}^{4} \bar{g}_{i j} \dot{u}^{j}+\sum_{j, k=0}^{4} \bar{\Gamma}_{i \mid j k} u^{j} u^{k}\right)+\lambda \sum_{j=0}^{3}\left(\frac{\partial A_{i}}{\partial x^{j}}-\frac{\partial A_{j}}{\partial x^{i}}\right) u^{j}+\dot{\lambda} A_{i}=0, \quad i=0, \ldots, 3 .
$$

Здесь $\bar{\Gamma}_{i \mid j k}$ - символы Кристоффеля симметричной римановой косвязности [2] на $M$, т.е.

$$
2 \bar{\Gamma}_{i \mid j k}=\frac{\partial \bar{g}_{k i}}{\partial x^{j}}+\frac{\partial \bar{g}_{i j}}{\partial x^{k}}-\frac{\partial \bar{g}_{j k}}{\partial x^{i}}, \quad i, j, k=0, \ldots, 4 .
$$

Мы будем рассматривать только такие тензорные поля на пятимерном многообразии $M$, которые удовлетворяют условию иилиндричности по пятой координате, т.е. не зависят от пятой координаты. Тогда пятое уравнение в силу условия цилиндричности имеет вид

$$
\frac{d}{d t}\left(m\left(-\kappa \sum_{j=0}^{3} A_{j} u^{j}+\bar{g}_{44} u^{4}\right)+\lambda\right)=0 .
$$

Следовательно,

$$
m\left(-\kappa \sum_{j=0}^{3} A_{j} u^{j}+\bar{g}_{44} u^{4}\right)+\lambda \equiv Z
$$

\footnotetext{
1) В римановой метрике кратчайшие.
} 
на $I$. Итак, пятая координата импульса $p_{4}$ является интегралом движения, и мы отождествляем ее с зарядом частицы. Как и в классической механике, существование интеграла движения (заряда частицы) обусловлено условием цилиндричности по соответствуюшей координате $\left(x^{4}\right)$. Отметим, что $\partial / \partial x^{4}$ есть соответствующее векторное поле Киллинга.

Здесь мы не будем подвергать вариации множитель Лагранжа $\lambda$, это будет сделано в разделе 4. Условие допустимости имеет вид

$$
\sum_{i=0}^{3} A_{i} u^{i}+u^{4}=0
$$

Из пятого уравнения Лагранжа получаем $\lambda=-m\left(\bar{g}_{44}+\kappa\right) u^{4}+Z$. Подставляя это выражение в первые четыре уравнения Лагранжа, получим уравнения допустимых геодезических

$$
\begin{gathered}
m\left(\sum_{j=0}^{3} \bar{g}_{i j} \dot{u}^{j}-\left(\bar{g}_{44}+2 \kappa\right) A_{i} \dot{u}^{4}+\sum_{j, k=0}^{3} \bar{\Gamma}_{i \mid j k} u^{j} u^{k}+\right. \\
\left.\quad+\sum_{j=0}^{3}\left(\bar{g}_{44}+2 \kappa\right)\left(\frac{\partial A_{j}}{\partial x^{i}}-\frac{\partial A_{i}}{\partial x^{j}}\right) u^{j} u^{4}\right)+ \\
+m\left(-\frac{1}{2} \frac{\partial \bar{g}_{44}}{\partial x^{i}}\left(u^{4}\right)^{2}-A_{i} \sum_{j=0}^{4} \frac{\partial \bar{g}_{44}}{\partial x^{j}} u^{j} u^{4}\right)+ \\
+Z \sum_{j=0}^{3}\left(\frac{\partial A_{i}}{\partial x^{j}}-\frac{\partial A_{j}}{\partial x^{i}}\right) u^{j}=0, \quad i=0, \ldots, 3 .
\end{gathered}
$$

Поскольку $\bar{g}_{44}=-2 \kappa$, эти уравнения совпадают с классическими уравнениями движения релятивистской частицы с зарядом $Z$ в электромагнитном поле, их можно также записать в виде

$$
\frac{\stackrel{*}{D} \gamma^{\prime}}{d t}+Z d A\left(\gamma^{\prime}, \cdot\right)=0
$$

Символом $\stackrel{*}{D} / d t$ мы обозначаем контравариантное дифференцирование вдоль пути $\gamma$ (контравариантная производная векторного поля является ковекторным полем, см. [2]). Символы Кристоффеля соответствуюшей косвязности вычисляются по формуле

$$
2 \Gamma_{i \mid j k}=\frac{\partial g_{k i}}{\partial x^{j}}+\frac{\partial g_{i j}}{\partial x^{k}}-\frac{\partial g_{j k}}{\partial x^{i}}, \quad i, j, k=0, \ldots, 3
$$

где $\left(g_{i j}\right)_{i, j=0, \ldots, 3}$ - метрический тензор на распределении $\mathbf{A}$. Поскольку этот тензор невырожден, мы можем поднять индекс $i$. Тогда уравнения движения примут классический вид. 


\section{3. ИНВАРИАНТНОСТЬ ТЕОРИИ}

3.1. При рассмотрении четырехмерного многообразия обшей теории относительности всегда требуют, чтобы все определенные на нем физические объекты были инвариантны по отношению к произвольным заменам координат. Для любой замены координат на четырехмерном многообразии (с общей гладкой структурой) ее матрица Якоби принадлежит группе $G L(4)$. На пятимерном гладком многообразии это требование приводит к тому, что все физические объекты должны быть $G L(5)$-инвариантны. Однако ни одно физическое уравнение не проявляет столь высокую инвариантность. По-видимому, мы должны ограничить гладкую структуру пятимерного многообразия так, чтобы включить в нее только те преобразования координат, относительно которых инвариантны классические уравнения движения.

В предыдущей работе [2] мы показали, что гладкая структура на многообразии связана с каузальной структурой на этом многообразии. Поэтому начнем с определения типа причинности в $T M$.

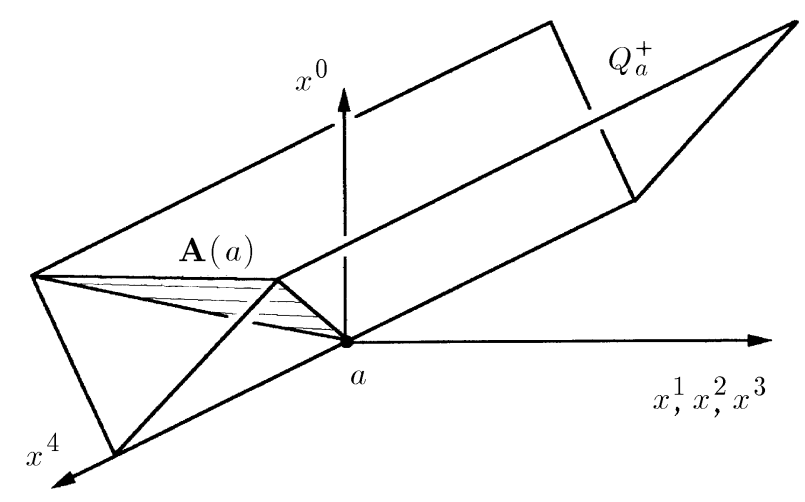

Известно, что 4-потенциал электромагнитного поля может принимать любые вещественные значения. Следовательно, гиперплоскость $\mathbf{A}(x):=\left\{u \in T_{x} M \mid n(x) \cdot u=0\right\}$ может поворачиваться как угодно в $T_{x} M$, но в силу определения нормали $n$ не может содержать ось $\partial_{4}$. Из физических соображений пересечение гиперплоскости $\mathbf{A}(x)$ с конусом будушего $Q_{x}^{+}$в $T_{x} M$ должно быть эллиптическим конусом размерности 4 . Следовательно, размерность конуса $Q_{x}^{+}$равна 5 и замыкание этого конуса содержит прямую $\operatorname{Lin}\left\{\partial_{4}\right\}$ (см. рисунок). Точнее, конус будушего $Q_{x}^{+}$является декартовым произведением прямой $\operatorname{Lin}\left\{\partial_{4}\right\}$ и эллиптического конуса. Последний задается с помошью сужения скалярного произведения $\langle\cdot, \cdot\rangle$ на линейное пространство $\mathbf{A}(x)$. По предположению это сужение является псевдоримановой метрикой сигнатуры $(+---)$.

3.2. Мы считаем допустимыми только те преобразования координат, которые сохраняют причинность. Такие преобразования образуют группу. При найденном нами типе причинности мы должны ограничиться группой вида 2.3.2 статьи [2], т.е. такой, что 
выполняется условие

$$
\frac{\partial y^{i}}{\partial x^{4}} \equiv 0, \quad i=0, \ldots, 3
$$

Следовательно, прямая $\operatorname{Lin}\left\{\partial_{4}\right\}$ является инвариантным подпространством. Координаты векторов из этого инвариантного подпространства преобразуются как одномерные векторы, т.е. умножаются на масштабный множитель

$$
u^{4} \frac{\partial}{\partial x^{4}}=u^{4} \frac{\partial y^{4}}{\partial x^{4}} \frac{\partial}{\partial y^{4}}
$$

Фиксируем масштаб вдоль пятой координаты: пусть

$$
\frac{\partial y^{4}}{\partial x^{4}} \equiv \pm 1
$$

(такие матрицы также образуют группу). Тогда координаты векторов из $\operatorname{Lin}\left\{\partial_{4}\right\}$ будут псевдоскалярами. Кроме того, поскольку мы рассматриваем только гладкие замены координат, то имеем

$$
\frac{\partial}{\partial x^{4}} \frac{\partial y^{i}}{\partial x^{j}}=\frac{\partial}{\partial x^{j}} \frac{\partial y^{i}}{\partial x^{4}} \equiv 0, \quad i, j=0, \ldots, 4 .
$$

Следовательно, условие иилиндричности $\partial v / \partial x^{4}=0$ для любого тензорного поля $v$ (физической величины) инвариантно.

3.3. Поскольку координаты ковекторов умножаются на транспонированную матрицу Якоби, отношение порядка в $T_{x}^{*} M$ (т.е. в $L^{*}$, см. п. 2.6 в [2]) также сохраняется. Инвариантным подпространством является $\operatorname{Lin}\left\{d x^{0}, d x^{1}, d x^{2}, d x^{3}\right\}$. Ковекторы из этого подпространства преобразуются как четырехмерные ковекторы. Пятая координата любого ковектора преобразуется как псевдоскаляр. Поэтому для ковектора импульса координату $p_{4}$ можно отождествить с зарядом частицы. При смене ориентации $x^{4}$ заряд меняет знак.

3.4. Распределение А было задано в координатах. Проверим его инвариантность. По определению $n_{4} \equiv+1$ (условие нормировки нормали). После замены координат пятая координата нормали перейдет в $n_{4} \partial x^{4} / \partial y^{4}$, т.е. умножится на \pm 1 . Будем рассматривать нормаль с точностью до ориентации: если преобразование координат меняет ориентацию $x^{4}$, то умножим нормаль на -1 . Определенная таким образом нормаль инвариантна с точностью до возможной смены ориентации при преобразованиях координат в $M^{5}$. Распределение $\mathbf{A}$ инвариантно без всяких оговорок. Если рассматривать только замены координат в инвариантном подпространстве в $L^{*}$, то координаты нормали преобразуются как компоненты четырехмерного ковектора. При произвольном преобразовании координат

$$
\sum_{i=0}^{3} A_{i} d x^{i}+d x^{4}=\sum_{j=0}^{3}\left(\sum_{i=0}^{3} A_{i} \frac{\partial x^{i}}{\partial y^{j}}+\frac{\partial x^{4}}{\partial y^{j}}\right) d y^{j}+\frac{\partial x^{4}}{\partial y^{4}} d y^{4}
$$


координаты нормали испытывают калибровочное преобразование. Следовательно, калибровочные преобразования являются следствием замены координат на $M^{5}$.

В результате замены координат $x \mapsto y$ ковектор нормали получает новые координаты

$$
\tilde{A}_{j}=\sum_{i=0}^{3} A_{i} \frac{\partial x^{i}}{\partial y^{j}}+\frac{\partial x^{4}}{\partial y^{j}}, \quad j=0, \ldots, 3
$$

(если ориентация координаты $x^{4}$ не меняется). Поэтому формула преобразования векторов базиса

$$
e_{i}=\frac{\partial}{\partial x^{i}}-A_{i} \frac{\partial}{\partial x^{4}}, \quad i=0, \ldots, 3,
$$

распределения А имеет вид

$$
e_{i}=\sum_{j=0}^{3} \frac{\partial y^{j}}{\partial x^{i}} \widetilde{e}_{j}
$$

где

$$
\widetilde{e}_{j}=\frac{\partial}{\partial y^{j}}-\tilde{A}_{j} \frac{\partial}{\partial y^{4}}, \quad j=0, \ldots, 3 .
$$

Калибровочные преобразования не проявляются в этой формуле, даже если они совершаются. В таком базисе рассматриваемое пространство скоростей “кажется" четырехмерным и имеюшим группу координатных преобразований $G L(4)$. В действительности группа преобразований координат сушественно больше.

Если ориентация $x^{4}$ меняется, то в соответствии с принятым соглашением знаки всех координат $A_{i}, i=0, \ldots, 3$, меняются на противоположные. Поэтому векторы базиса распределения $\mathbf{A}$ вообще не меняются.

3.5. Проверим инвариантность условия $g_{44}=-2 \kappa$ (определяюшего нормировку метрического тензора). Имеем

$$
\widetilde{g}_{44}=\sum_{i, j=0}^{4} g_{i j} \frac{\partial x^{i}}{\partial y^{4}} \frac{\partial x^{j}}{\partial y^{4}}=g_{44}\left(\frac{\partial x^{4}}{\partial y^{4}}\right)^{2}=g_{44}
$$

т.е. условие $g_{44}=$ const инвариантно.

3.6. Аналогично проверяется, что при преобразовании координат $x \mapsto y$ проекция $\mathrm{pr}$ на распределение А преобразуется следуюшим образом:

$$
\mathrm{pr}=\sum_{k=0}^{3} e_{k} \otimes d x^{k}=\sum_{k=0}^{3} \widetilde{e}_{k} \otimes d y^{k}
$$

где $\widetilde{e}_{k}$ определено в п. 3.4. Следовательно, матрица проекции pr в новых координатах имеет вид

$$
P=\left(I_{4} \mid\left(-\tilde{A}_{j}\right)_{j=0, \ldots, 3}\right)
$$

( $\tilde{A}_{j}$ определено в п. 3.4$)$. 
3.7. Итак, построенная нами теория инвариантна под действием группы диффеоморфизмов $L \rightarrow L$, матрица Якоби которых имеет вид

$$
\left(\begin{array}{cccc} 
& & & 0 \\
& * & & \vdots \\
& & & 0 \\
* & \ldots & * & \pm 1
\end{array}\right) .
$$

Левый верхний блок (квадратный, размерности 4) соответствует невырожденным линейным преобразованиям первых четырех координат. Левый нижний блок соответствует калибровочнымм преобразованиям первых четырех координат ковектора нормали к распределению $\mathbf{A}$, т.е. 4-потенциала электромагнитного поля. Число в правом нижнем блоке соответствует смене знака заряда с одновременной сменой ориентации нормали (смене знака 4-потенциала). Отметим, что все классические физические уравнения инвариантны по отношению к этому (дискретному) преобразованию.

\section{4. УРАВНЕНИЯ ДВИЖЕНИЯ ПРОБНОЙ ЧАСТИЦЫ}

4.1. В этом разделе мы дадим бескоординатный вывод уравнений движения пробной частицы, т.е. частицы, которая сама не возмушает электромагнитное и гравитационное поля.

Рассмотрим внешнюю производную от ковектора нормали $n$ как от 1-формы:

$$
F:=d n=\sum_{\substack{i, j=0, i<j}}^{3}\left(\frac{\partial A_{j}}{\partial x^{i}}-\frac{\partial A_{i}}{\partial x^{j}}\right) d x^{i} \wedge d x^{j}
$$

где мы использовали условие цилиндричности по $x^{4}$. По построению $F$ есть 2-форма на $T M^{5}$, но у нее отличны от нуля только первые четыре компоненты. Форма $F$ является тензором напряженности электромагнитного поля.

Распределение $\mathbf{A}$ можно задать также с помошью векторных полей $e_{k}:=\partial_{k}-A_{k} \partial_{4}$, $k=0, \ldots, 3$. Очевидно, что $\mathbf{A}(x)=\operatorname{Lin}\left\{e_{k}(x) \mid k=0, \ldots, 3\right\} \forall x \in M^{5}$. С помощью теоремы Фробениуса легко установить, что распределение $\mathbf{A}$ не интегрируемо (вполне неголономно): $\left[e_{i}, e_{j}\right]=-F_{i j} \partial_{4}$. Распределение $\mathbf{A}$ голономно тогда и только тогда, когда электромагнитное поле отсутствует.

4.2. Уравнения допустимых геодезических можно выводить, основываясь либо на формуле первой вариации действия (длины), либо на формуле первой вариации энергии. Эти подходы эквивалентны, поэтому мы выберем второй путь.

ОПреДЕлЕниЕ. Пусть $m \in \mathbf{R} \backslash\{0\}, \lambda: I \rightarrow \mathbf{R}-C^{1}$-гладкая функция, $\gamma: I \rightarrow M^{5}-$ (кусочно)-гладкий путь. Число

$$
E(\gamma, \lambda):=\frac{m}{2} \int_{I}\left\langle\gamma^{\prime}(t), \gamma^{\prime}(t)\right\rangle d t+\int_{I} \lambda(t) n(\gamma(t)) \cdot \gamma^{\prime}(t) d t
$$


называется энергией пути $\gamma$ при условии $\lambda$.

Ясно, что для допустимых путей энергия от $\lambda$ не зависит ( $\lambda$ - множитель Лагранжа).

Пусть $\sigma: I \times(-\varepsilon, \varepsilon) \rightarrow M^{5}$ - гладкая вариация пути $\gamma=\sigma(\cdot, 0), \varepsilon>0$. Пусть $\mu: I \times$ $(-\varepsilon, \varepsilon) \rightarrow \mathbf{R}$ - гладкая вариация функции $\lambda=\mu(\cdot, 0)$, согласованная с вариацией пути, т.е. существует такая функция $f: M^{5} \rightarrow \mathbf{R}$, что $\mu=f \circ \sigma$. Возникает функция $\tau \mapsto$ $E(\sigma(\cdot, \tau), \mu(\cdot, \tau))$. Найдем

$$
\delta E(\tau):=\frac{d E(\sigma(\cdot, \tau), \mu(\cdot, \tau))}{d \tau} .
$$

При достаточной гладкости метрического тензора и распределения $\mathbf{A}$ очевидно, что

$$
\delta E(\tau)=m \int_{I} \frac{\stackrel{*}{D} \sigma_{t}^{\prime}(t, \tau)}{d \tau} \cdot \sigma_{t}^{\prime}(t, \tau) d t+\int_{I} \frac{\partial\left(\mu(t, \tau) n(\sigma(t, \tau)) \cdot \sigma_{t}^{\prime}(t, \tau)\right)}{\partial \tau} d t
$$

(использована симметричная риманова косвязность). Введем векторные поля вдоль отображения $\sigma$ :

$$
X:=d \sigma\left(\frac{\partial}{\partial t}\right)
$$

- продольное векторное поле и

$$
Y:=d \sigma\left(\frac{\partial}{\partial \tau}\right)
$$

- поперечное векторное поле.

Лемма 1. Если вариация $\sigma C^{2}$-гладкая и косвязность симметрична, то

$$
\frac{\stackrel{*}{D} X}{\partial \tau}=\frac{\stackrel{*}{D} Y}{\partial t}
$$

Отсюда следует, что

$$
\frac{\partial}{\partial t}\langle X, Y\rangle=\frac{\stackrel{*}{D} X}{\partial t} \cdot Y+\frac{\stackrel{*}{D} X}{\partial \tau} \cdot X .
$$

Лемма 2. Пусть $\sigma \in C^{2}, \quad \mu \in C^{1}, \quad n \in C^{1}$ и существует функиия $f: M^{5} \rightarrow \mathbf{R}$ такая, что $\mu=f \circ \sigma$. Тогда

$$
\frac{\partial((\mu n \circ \sigma) \cdot X)}{\partial \tau}=d(f n)(Y, X)+\frac{\partial((\mu n \circ \sigma) \cdot Y)}{\partial t} .
$$

Итак,

$$
\delta E(\tau)=\left.m\langle X, Y\rangle\right|_{t=a} ^{t=b}-m \int_{I} \frac{*}{D X} \partial \underline{\partial t} \cdot Y d t+\left.(\mu n \circ \sigma) \cdot Y\right|_{t=a} ^{t=b}+\int_{I} d(f n)(Y, X) d t
$$

где $I=[a, b]$. 
Лемма 3. Если $f: U \rightarrow \mathbf{R}-C^{1}$-гладкая функиия, $n-C^{1}$-гладкое ковекторное поле на области $U \subset M^{5}, \operatorname{mo} d(f n)=(d f) \wedge n+f d n$.

Будем считать, что вариация проводится при закрепленных концах. Получаем формулу первой вариации энергии

$$
\begin{aligned}
& \delta E(\tau)=-m \int_{I} \frac{\stackrel{*}{D} X}{\partial t} \cdot Y d t+\int_{I}\left(\mu d n(Y, X)-\frac{\partial \mu}{\partial t} n \cdot Y+\frac{\partial \mu}{\partial \tau} n \cdot X\right) d t \\
& \delta E(0)=-m \int_{I} \frac{\stackrel{*}{D} \gamma^{\prime}}{\partial t} \cdot Y d t+\int_{I}\left(\lambda d n\left(Y, \gamma^{\prime}\right)-\frac{\partial \lambda}{\partial t} n \cdot Y\right) d t+\left.\int_{I} \frac{\partial \mu}{\partial \tau}\right|_{\tau=0} n \cdot \gamma^{\prime} d t .
\end{aligned}
$$

В силу произвольности векторных полей $Y,\left.\frac{\partial \mu}{\partial \tau}\right|_{\tau=0}$, получаем уравнения движения

$$
m \frac{\stackrel{*}{D} \gamma^{\prime}}{d t}+\frac{d \lambda}{d t} n+\lambda d n\left(\gamma^{\prime}, \cdot\right)=0
$$

и условие допустимости $n \cdot \gamma^{\prime}=0$.

ЛЕмма 4. Если метрический тензор удовлетворяет условию иилиндричности по координате $x^{4}$, то для символов Кристоффеля симметричной римановой косвязности имеет место соотношение $\Gamma_{k \mid i 4}=-\Gamma_{i \mid k 4}, i, k=0, \ldots, 4$.

Утверждение леммы следует из симметричности метрического тензора на $M^{5}$. В бескоординатной записи это соотношение имеет вид

$$
\left(\stackrel{*}{\nabla}_{u} \partial_{4}\right) \cdot v=-\left(\stackrel{*}{\nabla}_{v} \partial_{4}\right) \cdot u
$$

для любых векторов $u, v$. В частности,

$$
\left(\nabla_{u}^{*} \partial_{4}\right) \cdot u=0
$$

для любого вектора $u$.

Запишем пятое из уравнений движения

$$
m \frac{\stackrel{D}{ }^{\prime}}{d t} \cdot \partial_{4}+\frac{d \lambda}{d t}=0
$$

Так как косвязность риманова, то по лемме 4

$$
\frac{d}{d t}\left(m\left\langle\gamma^{\prime}, \partial_{4}\right\rangle+\lambda\right)=m\left(\frac{\stackrel{*}{D} \gamma^{\prime}}{d t} \cdot \partial_{4}+\left(\stackrel{*}{\nabla}_{\gamma^{\prime}} \partial_{4}\right) \cdot \gamma^{\prime}\right)+\frac{d \lambda}{d t}=0
$$


Обозначим $Z:=m\left\langle\gamma^{\prime}, \partial_{4}\right\rangle+\lambda$. Мы доказали, что $Z=$ const вдоль пути $\gamma$. Отождествим $Z$ с зарядом частицы, как и в разделе 2 . Тогда первые четыре уравнения движения можно переписать в виде

$$
m \frac{\stackrel{*}{D} \gamma^{\prime}}{d t}+Z d n\left(\gamma^{\prime}, \cdot\right)=0
$$

Эти уравнения инвариантны, т.к. ковекторное поле в левой части равенства всегда принадлежит инвариантному четырехмерному подпространству кокасательного расслоения (см. п. 3.3). Здесь $\stackrel{*}{D} \gamma^{\prime} / d t$ обозначает дифференцирование пути $\gamma$ с помошью косвязности, получающейся ограничением симметричной римановой косвязности на многообразии $M$ на первые четыре координатные функции. Она совпадает с симметричной римановой косвязностью метрического тензора $\left(g_{j k}\right)_{j, k=0, \ldots, 3}$, т.е. сужения скалярного произведения $\langle\cdot, \cdot\rangle$ на распределение $\mathbf{A}$. Итак, уравнения допустимых геодезических на рассматриваемом нами четырехмерном распределении совпадают с классическими уравнениями движения заряженной частищы общей теории относительности.

Пятая координата вектора $\gamma^{\prime}$ в эти уравнения не входит. Она определяется из условия допустимости $n \cdot \gamma^{\prime}=0$.

Итак, если сужение метрического тензора на распределение $\mathbf{A}$ невырождено и $m \neq 0$, то при фиксированной начальной точке $\gamma(0) \in M$, векторе скорости $\gamma^{\prime}(0) \in T_{\gamma(0)} M$ и заряде $Z \in \mathbf{R}$ задача Коши для допустимых геодезических имеет единственное решение, определенное на некотором промежутке $[0, \varepsilon), \varepsilon>0$. Это решение гладкое (в силу гладкости метрического тензора и распределения $\mathbf{A}$ ) и гладко зависит от начальных данных.

4.3. СлеДСТвиЕ. Если $m \neq 0$, то при движении вдоль допустимой геодезической норма вектора скорости сохраняется.

Действительно,

$$
\frac{d}{d t}\left\langle\gamma^{\prime}, \gamma^{\prime}\right\rangle=2 \frac{*}{D \gamma^{\prime}} d \gamma^{\prime}=\frac{2}{m}\left(m \frac{*}{D \gamma^{\prime}} d t \gamma^{\prime}+\frac{d \lambda}{d t} n \cdot \gamma^{\prime}+\lambda d n\left(\gamma^{\prime}, \gamma^{\prime}\right)\right)=0
$$

Мы доказали, что на рассматриваемом нами распределении, как и в обшей теории относительности, допустимые геодезические бывают трех сортов: временные $\left(\left|\gamma^{\prime}\right|>0\right)$, световые $\left(\left|\gamma^{\prime}\right|=0\right)$ и пространственные $\left(\left|\gamma^{\prime}\right|<0\right)$. Поскольку вектор скорости всегда принадлежит распределению $\mathbf{A}$, эта норма ничем не отличается от нормы в четырехмерном псевдоримановом многообразии.

Отсюда следует также, что формула первой вариации энергии совпадает с формулой первой вариации длины (действия) при условии, что все продольные линии вариации параметризованы длиной дуги. Для функционала энергии это условие излишне.

4.4. Красивым свойством данной теории является то, что калибровочные преобразования оказываются заменами координат на пятимерном многообразии. Этим свойством обладают также теории Калуцы-Клейна, но в этом классе теорий на пятимерном многообразии не возникает каузальной структуры, т.к. в них используется только один 
класс геодезических. В нашей теории имеются все три класса геодезических (временные, световые и пространственные), поэтому предлагаемая в настоящей работе теория не эквивалентна теориям типа Калуцы-Клейна.

4.5. Отметим, что предложенная теория не эквивалентна теории Вейля $[7,8]$. В теории Вейля распределение связности задано на $T P$, где $P$ - расслоенное пространство $P\left(M^{4}, U(1)\right)$. Это очень близко к нашему подходу. Однако в теории Вейля распределение связности определяется первым структурным уравнением Картана и, следовательно, зависит от поля метрического тензора. В нашей теории распределение $\mathbf{A}$ не зависит от метрики, а зависит только от электромагнитного поля. Кроме того, использование специальной гладкой структуры позволяет нам не делать априори никаких предположений о природе слоя $\left(S^{1}\right.$ или $\left.R^{1}\right)$.

В модели Вейля также сушественно используется то обстоятельство, что слой является группой Ли. Сравнительно недавно этот подход был обобшен на случай, когда слой является однородным пространством [9]. В нашей теории структура однородного пространства на слоях не используется, что делает предлагаемую модель потенциально более общей.

\section{Список литературы}

[1] V. Gromov. Carnot-Carathéodory spaces seen from within. Preprint IHES/M/94/6. Bures-Sur-Yvette: Institut des Hautes Etudes Scientifiques, 1994.

[2] В. Р. Крым. ТМФ. 1999. Т. 119. № 2. С. 264-281.

[3] Л. С. Понтрягин, В. Г. Болтянский, Р. В. Гамкрелидзе, Е. Ф. Мищенко. Математическая теория оптимальных процессов. М.: ГИФМЛ, 1961.

[4] Н. Н. Петров. Вестн. СПбГУ. Сер. 1. 1993. № 3. С. 28-32.

[5] R. Montgomery. J. Dynam. Control Systems. 1995. V. 1. P. 49-90.

[6] R. Montgomery. Progr. Math. 1996. V. 144. P. 325-339.

[7] N. Straumann. Early History of Gauge Theories and Weak Interactions. In: Invited Talk at the PSI Summer School on Physics with Neutrinos, Zuoz, Switzerland, 1996; hep-ph/9609230, http://xxx.lanl.gov.

[8] H. Weyl. Z. Phys. 1929. V. 56. P. 330-352.

[9] R. Coquereaux, A. Jadczyk. Commun. Math. Phys. 1983. V. 90. P. 79-100.

Поступила в редакцию 11.III.1998 г., после доработки 8.IX.1998 г. 